\title{
La indagación como herramienta de enseñanza en el museo de ciencias naturales: Un estudio de caso acerca del fortalecimiento de las prácticas de guianza
}

\author{
Miguel Angel Alzate Agudelo (D), Marlenny Guevara Guerrero (iD \\ Centro de Investigaciones en Psicología, Cognición y Cultura, Instituto de Psicología, Universidad del Valle. \\ Cali.Colombia.miguel.alzate@correounivalle.edu.co,marlenny.guevara@correounivalle.edu.co
}

[Recibido: 3 agosto 2020. Revisado: 31 enero 2021. Aceptado: 29 abril 2021]

\begin{abstract}
Resumen: Los museos son contextos privilegiados para el aprendizaje por medio de la indagación, donde se favorecen las interacciones entre guías-exhibiciones-visitantes. Este estudio presenta un proceso de intervención con los 4 guías del Museo Departamental de Ciencias Naturales de Cali, Colombia, en el cual se observó y analizó su interacción con niños visitantes del museo $(\mathrm{N}=1135$, Edad = entre los 5 y 13 años), procedentes de 28 instituciones educativas de la región. El estudio empleó un diseño mixto pre-post sin grupo control, un seguimiento microgenético de 7 meses y uso de video-feedback. Los resultados de la intervención revelaron cambios positivos en las prácticas de guianza, tales como: exploración de expectativas e ideas previas de los niños, aumento y diversificación de las preguntas, y aumento de preguntas abiertas. Adicionalmente, durante la fase de intervención, se registró un aumento en el uso de verbalizaciones complejas (i. e. descripciones, predicciones y explicaciones) por parte de los niños visitantes. Estos hallazgos contribuyen al campo de lainvestigación en los museos, al fortalecer tanto las prácticas de guianza como favorecer el desarrollo dehabilidades de pensamiento en los niños visitantes
\end{abstract}

Palabras clave: museos; indagación; prácticas de guianza; habilidades de pensamiento científico; video-feedback.

Inquiry as a teaching tool in the museum: A case study on strengthening guidance practices

Abstract: Museums are privileged contexts for learning through inquiry, fostering interactions between guidesexhibits-visitors. This study presents an intervention process with the four guides of the Departmental Museum of Natural Sciences in Cali, Colombia. The observation and analysis of the interaction of the guides with the visiting children of the museum $(\mathrm{N}=1135$, Age $=$ between 5 and 13 years), coming from 28 educational institutions of the region were carried out. A pre-post design without a control group and a 7-month microgenetic follow-up using video feedback were implemented. The intervention process results revealed positive changes in guiding practices, such as the exploration of children's expectations and previous ideas, increase and diversification of questions, and an increase of open questions. Moreover, during the tours, more complex verbalizations were used by the museum visiting children during the intervention phase (i.e. descriptions, predictions, and explanations). These findings contribute to the field of research in museums by strengthening both guiding practices and favoring the development of thinking skills in visiting children.

Keywords: museums; inquiry; guiding practices; scientific thinking skills; video-feedback.

Para citar este artículo: Alzate M. A. y Guevara M. (2021) La indagación como herramienta de enseñanza en el museo de ciencias naturales: Un estudio de caso acerca del fortalecimiento de las prácticas de guianza. Revista Eureka sobre Enseñanza y Divulgación de las Ciencias 18(3), 3103. doi: 10.25267/Rev_Eureka_ensen_divulg_cienc.2021.v18.i3.3103

\section{Introducción}

Los museos son lugares privilegiados para el aprendizaje. Preguntas como “¿por qué será que el pelo de ciertos animales es diferente?, ¿cómo se forman los meteoritos? o ¿por qué las aves tienen diferentes formas de picos?"1 son ejemplos de las inquietudes que los niños ${ }^{2}$ manifiestan a los guías al visitar un museo de ciencias naturales. Esta curiosidad espontánea es el escenario propicio que emplean los guías del museo para

\footnotetext{
${ }^{1}$ Verbalizaciones de niños durante visitas escolares al Museo Federico Carlos Lehman, Cali, Colombia.

${ }^{2}$ Se empleará el término "niños" para referirnos tanto a niños como niñas.
} 
incentivar el aprendizaje durante los recorridos. Desde una pedagogía de la indagación (como referencia a Paulo Freire y Antonio Faundez), en palabras de Ordoñez (2020), el rol del educador consistiría en entender estas preguntas de los niños como oportunidades para promover su curiosidad científica.

Los museos de ciencias son reconocidos como espacios educativos informales, debido a la flexibilidad de los procesos de enseñanza que emergen durante los recorridos museales y que promueven el aprendizaje de las ciencias fuera del entorno escolar (Bell et al. 2016; Stocklmayer, Rennie y Gilbert 2010). En los cuales, al considerarse contextos de aprendizaje de libre elección, se vinculan los contenidos expuestos en las exhibiciones, con los intereses, experiencias de vida e ideas previas de los visitantes (Falk, Storksdieck y Dierking 2007). Usualmente, los museos de ciencias pueden dar lugar a experiencias que van desde lo "semi-informal" tales como las visitas escolares guiadas (correspondientes a los casos analizados en el presente estudio), hasta experiencias poco estructuradas tales como la visita de un grupo de turistas o una familia sin un propósito educativo aparente (Sacco, Falk y Bell 2014).

A partir del diseño de las exhibiciones, el guion museográfico y acompañamiento de los guías, los museos permiten a los visitantes "manipular, probar, explorar, predecir, cuestionar, observar y dar sentido al mundo natural y físico" (Sacco et al. 2014 p.1), al igual que interactuar directa o indirectamente con elementos que generalmente son ajenos a su cotidianidad (Sánchez 2013). Los museos se consideran contextos que permiten fomentar procesos de indagación, donde los niños visitantes pueden corroborar o contrastar los contenidos curriculares de la escuela y sus ideas previas, con evidencia tangible o información de "primera mano" disponible en las exhibiciones, que generalmente no se encuentra en las escuelas (Akamca, Yildirim y Ellez 2017; Glick y Samarapungavan 2008), además de permitir la asociación de conceptos y el desarrollo de habilidades de resolución de problemas (Ampartzaki, Kypriotaki, Voreadou, Dardioti y Stathi 2013).

Estas experiencias de aprendizaje que pueden ofrecer los museos, configuran un complejo ambiente de interés investigativo. Por ejemplo, estudios que examinan el potencial de los recorridos guiados para promover en los visitantes el interés por las ciencias (Guisasola y Morentin 2005; Silveira 2016), la relación entre escuela y museo (Aguirre 2013; Morentin y Guisasola 2014; Stocklmayer et al. 2010), el diseño de actividades museales basadas en la indagación (Ash y Klein 2000; Pérez y Chamizo 2011), entre otros. En particular, el presente estudio se centra en analizar las prácticas pedagógicas de los guías, que denominaremos prácticas de guianza, en interacción con los niños visitantes del museo.

Los museos donde se implementan procesos de guianza tradicional, se caracterizan por la poca frecuencia y bajo nivel de complejidad de las preguntas formuladas por los guías. Centran su oferta en experiencias contemplativas, que conllevan a la baja participación de los visitantes (Roldán, Ulloa, Vargas, Chura y Pacheco 2017). Dichas características también son comunes en contextos escolares, orientados a mantener el control de los grupos, donde se privilegian aspectos de repetición de la información y manejo memorístico de conocimientos de tipo factual, más que la comprensión de contenidos curriculares (Lee y Kinzie 2011; Smart y Marshall 2013; van Vondel, Steenbeek, van Dijk y van Geert 2017a).

$\mathrm{Al}$ concebir a los museos como espacios complementarios a la práctica educativa formal, el presente estudio explora las prácticas de guianza y participación de los niños visitantes (modalidad de visita escolar), durante los recorridos observados antes, durante y después de la implementación de una intervención basada en el uso de la indagación. Específicamente, el objetivo es caracterizar y fortalecer las prácticas de guianza en el Museo Departamental de Ciencias Naturales Federico Carlos Lehmann $(\mathrm{FCL})^{3}$ Cali, Colombia, a partir de un proceso de intervención que además promueva el desarrollo de habilidades de pensamiento científico en los niños visitantes (i.e. descripción, predicción y explicación) durante los recorridos en el museo.

\footnotetext{
${ }^{3}$ El museo FCL cuenta con la misión de generar conciencia ambiental en la población. Las exhibiciones siguen una línea temática que le permite a los visitantes "viajar" desde el espacio exterior hasta las profundidades de los ecosistemas marinos de la región.
} 


\section{Las prácticas de guianza como parte de un sistema: Guías - exhibiciones - visitantes del museo}

La perspectiva teórica de los sistemas dinámicos permite comprender fenómenos complejos al explicar las interacciones de los componentes del fenómeno en estudio y sus procesos de cambio en un periodo de tiempo (van Geert 2011). Esta teoría, adaptada de las ciencias de la Física y las Matemáticas a la Psicología, ha permitido entender el desarrollo y el aprendizaje como procesos dinámicos y variables (Steenbeck y van Geert 2013), en la cual se retoman los diversos elementos que hacen parte del sistema observado (Perinat, Lalueza y Sadurní 2007).

En nuestro estudio, consideramos propicia esta perspectiva para analizar y comprender las prácticas de guianza. Las preguntas de los guías y las respuestas de los visitantes no tienen lugar de manera aislada. Tanto guías como visitantes del museo, interactúan y se retroalimentan mutuamente al ser agentes activos de aprendizaje. En este estudio, las interacciones se entienden como relaciones bidireccionales que emergen en tiempo real y fluctúan de acuerdo con las demandas del contexto $^{4}$ (Figura 1).

Consideramos las prácticas de guianza como interacciones recíprocas, como un

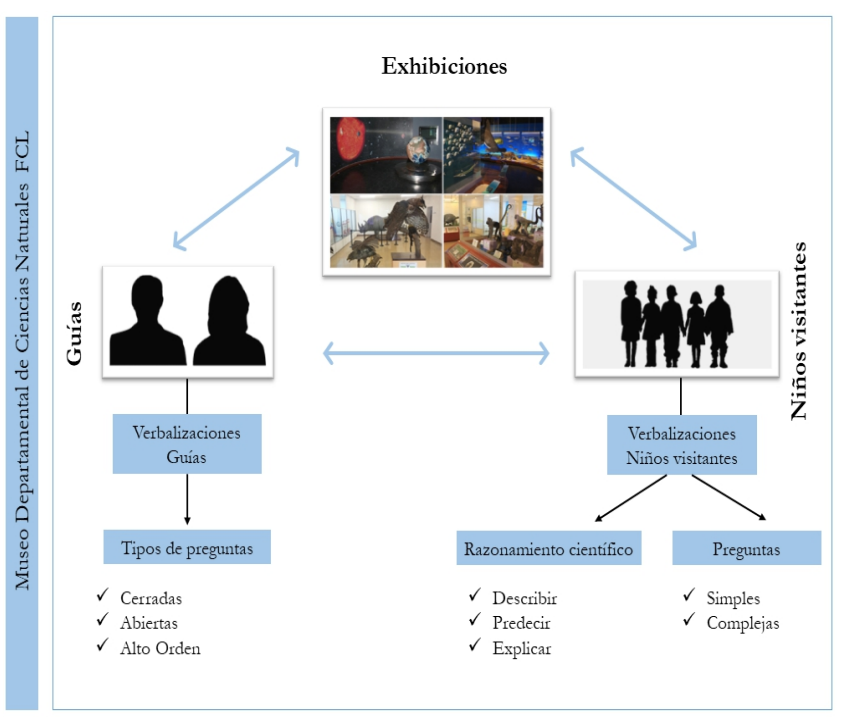

Figura 1. Interacción guías - exhibiciones - niños visitantes. visitantes del museo, en la medida en que las preguntas que formula el guía durante el recorrido por las exhibiciones, fomentan en los visitantes el uso de habilidades de pensamiento científico. Por su parte, los visitantes del museo y sus ideas previas se ponen en relación con los contenidos de las exhibiciones para responder a las preguntas del guía, y este a su vez genera nuevas explicaciones. Así mismo, las exhibiciones contienen información y características propias que estimulan la curiosidad y el razonamiento tanto del guía como de los visitantes del museo.

\section{El desarrollo de habilidades de pensamiento científico a partir de la indagación}

A partir de la indagación es posible promover el desarrollo de habilidades de pensamiento complejo, tales como las habilidades de pensamiento científico (i.e. descripción, predicción, explicación, etc.). Estas últimas son un conjunto de estrategias cognitivas que le permiten al individuo dar respuesta a preguntas acerca de cómo funciona el mundo, mediante la observación sistemática de los fenómenos que hacen parte de su cotidianidad (Morris, Croker, Masnick, Zimmerman 2012). Richard Feynman (1981, citado por Furman 2016), premio nobel de física, define el pensamiento científico como el placer de descubrir las cosas, como referencia a una actitud investigadora que implica la capacidad de realizar preguntas sobre las cosas que no se conocen y buscar posibles explicaciones. Esta idea de Feynman se relaciona con el concepto de curiosidad cientifica, igualmente abordado por dicho autor, un rasgo psicológico de dominio general a partir del cual se entiende que el comportamiento exploratorio, dirigido a la adquisición de nuevo conocimiento, es una característica psicológica espontánea y presente en el pensamiento cotidiano (Galvis y Concha 2018; Ordoñez 2020).

El aprendizaje por indagación implica el uso de prácticas investigadoras, tales como la formulación de preguntas (Loyens y Rikers 2011), que promueven la resolución de problemas, el uso de descripciones,

\footnotetext{
${ }^{4}$ Esta perspectiva ha sido empleada en estudios previos con contextos escolares. Ver van Vondel et al. $2017 \mathrm{a}$.
} 
predicciones, explicaciones, entre otras habilidades (Kuhn, 2011). Si bien esta ha sido una estrategia ampliamente usada en contextos educativos formales, cada vez es más utilizada en entornos como los museos. Estudios como el de Ash y Klein (2000), afirman que la indagación en museos brinda a los visitantes la oportunidad de comprender la ciencia a partir de las habilidades que la práctica científica demanda. Un ejemplo de indagación en museos se ilustra en el estudio de Roldán et al. (2017), mediante la implementación de estrategias como los senderos de indagación (SI), común en varios países de Latinoamérica. Según estos autores, los SI implementan ciclos de indagación (o el ciclo empírico) que consisten en plantear preguntas, observar el fenómeno y llegar a una reflexión acerca del mismo. Esta articulación entre el aprendizaje por indagación y las habilidades de pensamiento científico en los museos, permite vincular a los visitantes con problemáticas reales que cobran sentido a la luz de sus intereses y experiencias (Aguirre, 2013).

\section{Prácticas de guianza y procesos de interacción en el museo}

Empleamos el término "prácticas de guianza" para hacer referencia al desempeño de los guías como agentes educativos en contextos informales, al orientar a un individuo o un grupo de personas a través de espacios diseñados con un propósito educativo. Las prácticas de guianza remiten así a las interacciones entre el guía y los visitantes del museo.

Tanto en contextos de aprendizaje formales como informales, la principal forma de interacción corresponde a la comunicación verbal. Así pues, dentro de los elementos del discurso, autores como Smart y Marshall (2013), Li y Arshad (2013), destacan la importancia de las preguntas del agente educativo como estrategia para lograr un diálogo efectivo, que motive a los aprendices a elaborar sus ideas con el fin de involucrarlos en pensamientos de alto orden.

Un ejemplo se encuentra en el estudio de Eberbach y Crowley (2017) en un jardín botánico. Estrategias conversacionales como hacer preguntas y relacionar las experiencias con lo observado, fomentaba la participación de los niños visitantes, y la comprensión de los conceptos abordados en el recorrido. van Schijndel y Raijmakers (2016), afirman que la comprensión de los niños en un museo se ve altamente afectada por el acompañamiento o no de un guía que proporcione una estructura y logre centrar su atención sobre determinados elementos de las exhibiciones. Por consiguiente, los diferentes tipos de explicaciones, preguntas o demás medios de guianza resultan de gran interés al tratar de comprender procesos de enseñanza-aprendizaje en contextos informales.

Briceño y Tafur (2011), señalan que en un proceso de guianza, ambos actores (guía - visitantes) deben tener un papel protagónico. Esto se manifiesta en la interacción, preguntas que generan y sostienen el diálogo, y la atención de los visitantes, para evidenciar sus ideas previas e incentivar la generación de hipótesis y argumentación. En consecuencia, se considera que las preguntas abiertas permiten explorar la curiosidad y promover las comprensiones de los visitantes (ver Hohenstein y Tran 2007).

\section{Método}

El estudio implicó un acompañamiento y seguimiento exhaustivo a las prácticas de guianza, con el objetivo de fortalecerlas e identificar cómo se modificaban en el tiempo (antes, durante y después de la intervención).

\section{Guías participantes}

El estudio de intervención se llevó a cabo con los 4 guías ${ }^{5}$ del Museo FCL (Cali-Colombia) en interacción con grupos de niños de los colegios visitantes (entre los 5 y los 13 años de edad), provenientes de 28 instituciones educativas (IEs) de la región, que asistieron al museo durante el periodo de observación. En la Tabla 1 se especifican las características de los guías participantes y en la Figura 2 se indica la procedencia geográfica de las IEs.

\footnotetext{
${ }^{5}$ Corresponde a la totalidad de guías del museo, el cual, al ser relativamente pequeño, sólo cuenta con cuatro guías.
} 
Tabla 1. Caracterización demográfica de los guías participantes.

\begin{tabular}{|l|l|l|l|l|}
\hline \multicolumn{1}{|c|}{ Código } & \multicolumn{1}{|c|}{ Sexo } & \multicolumn{1}{|c|}{ Edad } & \multicolumn{1}{c|}{$\begin{array}{c}\text { Nivel de } \\
\text { estudios }\end{array}$} & Tiempo de experiencia como guía \\
\hline AJ & M & 64 & Bachiller & 6 años \\
\hline BL & F & 35 & Tecnólogo & 5 años \\
\hline DC & F & 21 & Tecnólogo & 3 meses \\
\hline CM & F & 24 & Tecnólogo & 2 meses \\
\hline
\end{tabular}

\section{Diseño del estudio}

Se implementó un diseño mixto tipo pre-post sin grupo control, con un seguimiento microgenético ${ }^{6}$ de 7 observaciones por cada guía a lo largo de 7 meses, en las cuales se registró su desempeño durante los recorridos. El estudio contó con 3 fases: La fase pretest, donde se grabaron 2 recorridos por guía. En la fase de intervención se implementaron 5 talleres con los guías y 3 sesiones de video-feedback, dirigidos por los investigadores. Los talleres abordaron temáticas como el rol del guía y niños visitantes durante los recorridos, importancia de la formulación de preguntas, espacios de participación, etc. Y las sesiones de video-feedback donde, a partir de preguntas formuladas por los investigadores, el guía observaba su desempeño durante un recorrido grabado previamente, y reflexionaba en torno a sus fortalezas y oportunidades de mejoramiento. Finalmente, durante la fase post-test, desarrollada mes y medio después de la intervención, se realizaron 2 grabaciones de recorridos por cada

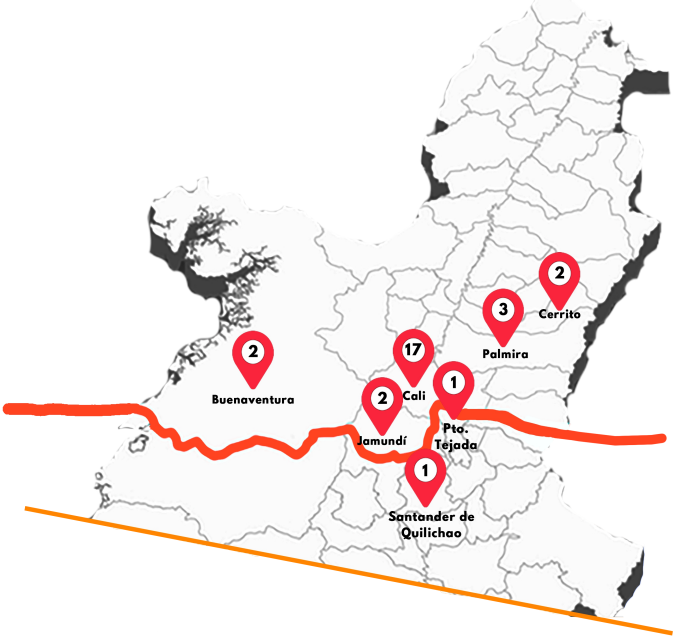

Figura 2. Cantidad de IEs visitantes del museo durante las observaciones, por lugares de procedencia. guía. Esto con el fin de comparar el antes y el después del desempeño de los guías, para finalmente identificar la presencia de cambios en sus prácticas de guianza sostenidos en el tiempo (Figura 3).

El registro fílmico de los recorridos del museo, se llevó a cabo con el consentimiento informado de los cuatro guías, las directivas del museo y los docentes de las IEs visitantes. El tiempo de grabación de los recorridos fue variable y comprendió entre 25 minutos a 1 hora y 20 minutos $^{7}$

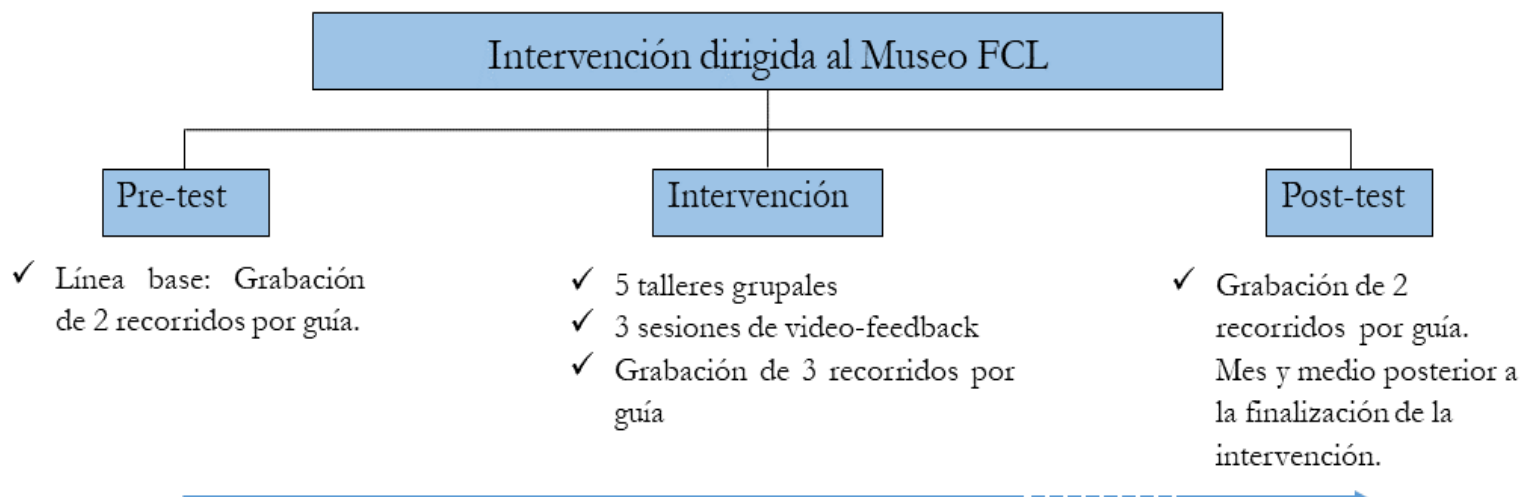

7 Meses

Figura 3. Fases del estudio.

${ }^{6}$ Consiste en realizar mediciones repetidas del fenómeno observado y así dar cuenta de cómo cambia la unidad de análisis en el tiempo (Siegler y Crowley 1991).

${ }^{7}$ Estas diferencias de tiempo se deben a variables externas al museo, propias de los grupos visitantes. 


\section{Implementación de la Intervención en el Museo FCL}

La intervención en el Museo FCL consistió en 5 talleres grupales y 3 sesiones individuales de videofeedback. Estos se enfocaron en promover procesos de indagación en los guías mediante el uso de preguntas y sensibilización en torno a prácticas de guianza que demandaran el uso de habilidades de pensamiento científico en los niños de los colegios visitantes. La Tabla 2 presenta la secuencia de actividades durante la intervención.

Tabla 2. Esquema de actividades desarrolladas en el proceso de intervención en el museo FCL.

\begin{tabular}{|c|c|c|}
\hline Actividad & Temática & Objetivos \\
\hline Taller 1 & $\begin{array}{l}\text { Potencial del } \\
\text { Museo y } \\
\text { Habilidades de } \\
\text { pensamiento } \\
\text { científico }\end{array}$ & $\begin{array}{l}\text { 1. Identificar las concepciones de los guías acerca del museo como } \\
\text { espacio de aprendizaje. } \\
\text { 2. Reflexionar sobre el potencial de los museos como contextos } \\
\text { educativos informales. } \\
\text { 3. Identificar las concepciones de los guías acerca de la presencia de } \\
\text { habilidades de pensamiento científico en niños. } \\
\text { 4. Reconocer la importancia de promover las habilidades de } \\
\text { pensamiento científico de niños en los museos. }\end{array}$ \\
\hline Taller 2 & $\begin{array}{l}\text { Indagación en el } \\
\text { Museo }\end{array}$ & $\begin{array}{l}\text { 1. Identificar el tipo de preguntas empleadas por los guías durante los } \\
\text { recorridos. } \\
\text { 2. Reconocer la importancia del uso de preguntas para promover el } \\
\text { desarrollo de habilidades de pensamiento en los visitantes del museo. } \\
\text { 3. Identificar y promover la formulación de diversos tipos de } \\
\text { preguntas durante la guianza. }\end{array}$ \\
\hline $\begin{array}{l}\text { Taller } 3 \text { (grupo } \\
\text { focal) }\end{array}$ & $\begin{array}{l}\text { El papel del guía y } \\
\text { el } \\
\text { niño en la } \\
\text { construcción de } \\
\text { conocimiento }\end{array}$ & $\begin{array}{l}\text { 1. Reflexionar en torno a prácticas de guianza centradas en los niños } \\
\text { visitantes. } \\
\text { 2. Integrar actividades de indagación para la interacción con los niños } \\
\text { visitantes. } \\
\text { 3. Identificar las habilidades de pensamiento científico promovidas } \\
\text { mediante la indagación. } \\
\text { 4. Introducción de la metodología del video-feedback. }\end{array}$ \\
\hline $\begin{array}{l}\text { Sesión de Video- } \\
\text { feedback } 1\end{array}$ & \multirow[t]{3}{*}{$\begin{array}{l}\text { Retroalimentación } \\
\text { individual }\end{array}$} & \multirow{3}{*}{$\begin{array}{l}\text { 1. Promover una reflexión crítica de cada guía acerca de sus prácticas } \\
\text { de guianza (Nota: se inició con videos del pre-test. Luego con videos } \\
\text { de recorridos realizados } 1 \text { semana previa a la sesión de video- } \\
\text { feedback). } \\
\text { 2. Identificar de manera conjunta los aspectos de mejora y estrategias } \\
\text { de acción para integrar en futuros recorridos guiados. }\end{array}$} \\
\hline $\begin{array}{l}\text { Sesión de Video- } \\
\text { feedback } 2\end{array}$ & & \\
\hline $\begin{array}{l}\text { Sesión de Video- } \\
\text { feedback } 3\end{array}$ & & \\
\hline Taller 4 & $\begin{array}{l}\text { Diseño inicial de } \\
\text { recorridos con } \\
\text { temáticas } \\
\text { específicas. }\end{array}$ & $\begin{array}{l}\text { 1. Generar una estructura inicial de un recorrido especializado. } \\
\text { 2. Propiciar un espacio para la práctica conjunta de los contenidos } \\
\text { abordados. }\end{array}$ \\
\hline Taller 5 & $\begin{array}{l}\text { Reflexión de cierre } \\
\text { ¿Cómo es un guía } \\
\text { ideal? }\end{array}$ & $\begin{array}{l}\text { 1. Establecer las fortalezas y oportunidades de mejoramiento de los } \\
\text { guías. } \\
\text { 2. Proporcionar a los guías retroalimentación sobre sus fortalezas } \\
\text { individuales. } \\
\text { 3. Evaluación general de la intervención. }\end{array}$ \\
\hline
\end{tabular}




\section{La retroalimentación basada en vídeo}

El método de video-feedback ha sido ampliamente utilizado como una herramienta para promover la reflexión autocrítica sobre desempeños de agentes educativos (Pennings, van Tartwijk, Wubbels, Claessens, van der Want, y Brekelmans 2014). Como estrategia de intervención basada en la evidencia, el video-feedback propicia cambios comportamentales en el agente educativo a partir de la observación de sus propias prácticas de enseñanza, donde obtiene retroalimentación en tiempo real (van Vondel, Steenbeek, van Dijk y van Geert 2017b). El uso del video-feedback resulta apropiado para este estudio porque favorece la reflexión de los guías sobre sus prácticas de

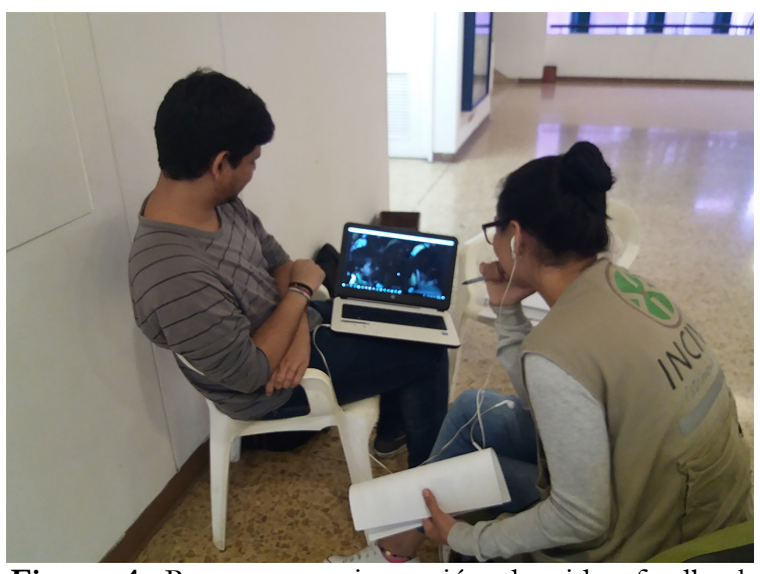

Figura 4. Recurso propio: sesión de video-feedback con una guía del museo FCL. guianza, y les permite identificar sus avances durante el proceso de intervención (Figura 4).

Las sesiones de video-feedback durante la intervención se llevaron a cabo con cada guía de la siguiente manera:

1. Selección de un video del proceso de guianza (investigadores): Se emplearon secuencialmente videos de recorridos obtenidos en el pre-test y durante la intervención.

2. Segmentación del video (investigadores): De cada video analizado se seleccionaron cuatro segmentos considerados relevantes por la interacción entre guías y niños visitantes (i.e. preguntas del guía y verbalizaciones de los niños visitantes).

3. Identificación de aspectos por fortalecer en las prácticas de guianza (investigadores): Se empleó una rejilla de observación para registrar el tipo de preguntas, retroalimentación y seguimiento a la comprensión de los niños visitantes, etc.

4. Observación del video junto con el guía para brindar retroalimentación de su proceso de guianza (investigadores y guía): Se propició la reflexión del guía sobre sus propias prácticas de guianza, por medio de preguntas evocadas por segmentos del video que evidenciaban fortalezas y aspectos de mejora. Por ejemplo: “¿cuál era el propósito de esta pregunta?”, "¿qué le indican estas respuestas de los niños visitantes acerca de la comprensión de la exhibición?”, “¿qué preguntas podría formular a los niños para establecer la relación entre X y Y elemento de las exhibiciones?, etc.

5. Registro de propuestas del guia para el fortalecimiento de sus prácticas de guianza (investigadores y guía): Se le pidió al guía que diligenciar en un formato, las fortalezas y modificaciones que propondría en su práctica de guianza.

\section{Plan de análisis}

$\mathrm{El}$ análisis de las prácticas de guianza se llevaron a cabo de manera intra-individual ${ }^{8}$ y grupal, al comparar los datos pre-post en las siguientes dimensiones: (1) características del perfil de las prácticas de guianza basado en la pauta de observación Classroom Assessment Scoring System (CLASS-Upper Elementary) correspondiente al dominio de apoyo instruccional. (2) el tipo de preguntas formuladas por los guías (i.e. Cerradas, abiertas y de alto orden), y (3) las verbalizaciones de los niños visitantes durante los recorridos guiados (i.e. preguntas cerradas, preguntas abiertas, descripciones, predicciones y explicaciones).

\footnotetext{
${ }^{8}$ Comparar varias mediciones del desempeño de un mismo guía en diferentes momentos
} 
La pauta de observación CLASS, comúnmente orientada a caracterizar el quehacer docente al interior del aula (Pianta, Hamre y Mintz 2012; Martínez, Godoy, Treviño, Varas y Fajardo 2018), es utilizada en el presente estudio para analizar y comparar las prácticas de guianza en el pre-test y post-test. En este estudio se empleó de manera cualitativa (nivel alto, medio, bajo) sólo el dominio de apoyo instruccional debido a que sus categorías son aplicables a contextos educativos informales y se relacionan estrechamente con la interacción del guía y los niños visitantes (Tabla 3).

Tabla 3. Dimensiones de análisis sobre apoyo instruccional de los guías a partir de pauta de observación CLASS.

\begin{tabular}{|c|c|c|c|c|}
\hline $\begin{array}{l}\text { Formatos de } \\
\text { aprendizaje } \\
\text { instruccional }\end{array}$ & $\begin{array}{l}\text { Comprensión del } \\
\text { contenido }\end{array}$ & $\begin{array}{c}\text { Análisis e } \\
\text { indagación }\end{array}$ & $\begin{array}{l}\text { Calidad de la } \\
\text { retroalimentación }\end{array}$ & $\begin{array}{c}\text { Diálogo } \\
\text { instruccional }\end{array}$ \\
\hline $\begin{array}{l}\text { Analiza las } \\
\text { modalidades o } \\
\text { estrategias que } \\
\text { involucran a los } \\
\text { niños participantes, } \\
\text { y aseguran un } \\
\text { proceso de } \\
\text { instrucción basado } \\
\text { en la indagación. } \\
\text { (Pianta et al. 2012). }\end{array}$ & $\begin{array}{l}\text { Se refiere tanto a la } \\
\text { profundidad de los } \\
\text { contenidos abordados } \\
\text { como a las estrategias } \\
\text { usadas para que los niños } \\
\text { comprendan esos } \\
\text { contenidos. Integran la } \\
\text { comprensión de los } \\
\text { hechos, las habilidades, } \\
\text { los conceptos y principios } \\
\text { de los contenidos. }\end{array}$ & $\begin{array}{l}\text { Evalúa el grado en } \\
\text { que los niños } \\
\text { participantes se } \\
\text { involucran en } \\
\text { habilidades de } \\
\text { pensamiento } \\
\text { complejo. }\end{array}$ & $\begin{array}{l}\text { Se refiere a } \\
\text { devoluciones acerca del } \\
\text { desempeño o esfuerzo } \\
\text { de los niños. Idealmente } \\
\text { se caracteriza por } \\
\text { intercambios de ida y } \\
\text { vuelta donde el adulto } \\
\text { responde y retoma las } \\
\text { preguntas y comentarios } \\
\text { de los participantes. } \\
\text { (Pianta et al. 2012). }\end{array}$ & $\begin{array}{l}\text { Consiste en el uso } \\
\text { intencional del diálogo } \\
\text { entre adulto y niños } \\
\text { respecto al contenido } \\
\text { presentado. Donde } \\
\text { estos últimos participan } \\
\text { activamente, y pueden } \\
\text { exponer sus preguntas y } \\
\text { comprensiones al } \\
\text { respecto. }\end{array}$ \\
\hline
\end{tabular}

Para el análisis de las verbalizaciones de los guías, se diseñó un esquema de codificación basado en las categorías planteadas por van Schijndel y Raijmakers (2016) sobre tipos de preguntas abiertas y cerradas, al igual que se adaptaron las categorías de Briceño y Tafur (2011), correspondientes a la tipología de las preguntas según su contenido. A cada tipo de pregunta formulada por el guía se le asignó una puntuación de 1 a 7 de acuerdo con su nivel de complejidad (Tabla 4).

Tabla 4. Esquema de codificación de las preguntas de los guías.

\begin{tabular}{|c|c|c|c|c|}
\hline Cód. & Categoría & Subtipo & Definición & Ejemplos \\
\hline 1 & \multirow{3}{*}{$\begin{array}{l}\text { Preguntas } \\
\text { cerradas }\end{array}$} & Verificación & $\begin{array}{l}\text { Se refiere al uso de repeticiones y } \\
\text { aspectos memorísticos. Busca } \\
\text { obtener la atención de los niños. }\end{array}$ & $\begin{array}{l}\text { "Chicos, yo les habia hablado de } 3 \text { clases de } \\
\text { tiburones que se manejan aquí en el Museo, } \\
\text { ¿Recuerdan cuáles son?" }\end{array}$ \\
\hline 2 & & Cotidianas & $\begin{array}{l}\text { Se refiere a hechos cotidianos. } \\
\text { Busca verificar ideas previas. }\end{array}$ & $\begin{array}{l}\text { "¿Han escuchado hablar del mono araña, de los } \\
\text { meteoritos? ¿Han ido al zoológico?" }\end{array}$ \\
\hline 3 & & Contenido & $\begin{array}{l}\text { Se refiere a conceptos y } \\
\text { definiciones. Busca explorar } \\
\text { vocabulario científico. }\end{array}$ & $\begin{array}{l}\text { "¿Qué temperatura hace en este ecosistema? } \\
\text { (páramos) ¿Cálido o frío? ¿Cómo se llaman los } \\
\text { animales que tienen huesos? ¿Qué animal es este?" }\end{array}$ \\
\hline 4 & \multirow{4}{*}{$\begin{array}{l}\text { Preguntas } \\
\text { abiertas }\end{array}$} & Verificación & $\begin{array}{l}\text { Se refiere a expectativas y opiniones. } \\
\text { Busca hacer seguimiento a la } \\
\text { participación durante las actividades. }\end{array}$ & $\begin{array}{l}\text { "¿Qué esperan encontrar en el museo? ¿Qué } \\
\text { opinión tienen acerca del vídeo que acabamos de } \\
\text { ver?" }\end{array}$ \\
\hline 5 & & Cotidianas & $\begin{array}{l}\text { Se refiere a situaciones comunes. } \\
\text { Busca verificar ideas previas } \\
\text { relacionadas con los contenidos. }\end{array}$ & $\begin{array}{l}\text { "¿Qué hacemos cuando tenemos mucho frío? } \\
\text { ¿Qué función creen que tienen los bomberos?" }\end{array}$ \\
\hline 6 & & Contenido & $\begin{array}{l}\text { Se refiere a conceptos y procesos. } \\
\text { Busca explorar la comprensión de } \\
\text { vocabulario científico y de contenido. }\end{array}$ & $\begin{array}{l}\text { "¿Por qué es importante el cóndor? ¿De dónde } \\
\text { creen que vienen los meteoros? ¿Qué creen que son } \\
\text { aves migratorias?" }\end{array}$ \\
\hline 7 & & Alto orden & $\begin{array}{l}\text { Se refiere a situaciones problema. } \\
\text { Busca generar cuestionamientos, } \\
\text { predicciones y explicaciones. }\end{array}$ & $\begin{array}{l}\text { "¿Por qué será que las ballenas no tienen sus hijos } \\
\text { allá en la Antártida? ¿Qué pasaría si el cóndor de } \\
\text { los andes no existiera? ¿Por qué el pelaje del oso } \\
\text { peręoso es distinto en ambos ecosistemas?” }\end{array}$ \\
\hline
\end{tabular}


Adicional a las verbalizaciones de los guías, se llevó a cabo el análisis de las verbalizaciones de niños de los colegios visitantes del museo. Para ello, se empleó el esquema de codificación propuesto en estudios previos (Guevara 2015; Guevara, van Dijk y van Geert 2016), y se asignó puntajes de 0 a 5 , de acuerdo con los niveles de complejidad de sus verbalizaciones. Adicionalmente, se clasificaron las preguntas formuladas por los niños como cerradas y abiertas (Tabla 5).

Tabla 5. Esquema de codificación de las verbalizaciones de los niños visitantes

\begin{tabular}{|c|c|c|c|}
\hline Cód. & Categoría & Definición & Ejemplos \\
\hline 0 & Otras verbalizaciones & $\begin{array}{l}\text { Verbalizaciones que no aplican } \\
\text { para las otras categorías. }\end{array}$ & Repeticiones, nombre de algún animal. \\
\hline 1 & Preguntas cerradas & $\begin{array}{l}\text { Preguntas que se responden con } \\
\text { sí/no, o son de nombramiento. }\end{array}$ & $\begin{array}{l}\text { "¿Ese animal es de verdad? ¿Qué es eso que está } \\
\text { ahi?" }\end{array}$ \\
\hline 2 & Preguntas abiertas & $\begin{array}{l}\text { Preguntas que indagan y } \\
\text { establecen relaciones entre } \\
\text { contenidos respecto a una } \\
\text { temática de interés. }\end{array}$ & $\begin{array}{l}\text { "¿Para qué le sirven las verrugas a la ballena? } \\
\text { ¿Por qué esa piedrita chiquita [meteorito] sería } \\
\text { capaz de matar a un ser humano?" }\end{array}$ \\
\hline 3 & Descripción & $\begin{array}{l}\text { Verbalizaciones que indican las } \\
\text { características de algo } \\
\text { observado. }\end{array}$ & $\begin{array}{l}\text { "El oso tiene mucho pelo. Tiene un color diferente } \\
\text { del pelaje. La estatura de los monos antiguos es } \\
\text { diferente a la de los monos americanos" }\end{array}$ \\
\hline 4 & Predicción & $\begin{array}{l}\text { Verbalizaciones que formulan } \\
\text { una situación hipotética sobre } \\
\text { una información brindada por } \\
\text { un otro. }\end{array}$ & $\begin{array}{l}\text { Guía: “¿Qué pasaría si no comiéramos en muchos } \\
\text { días?" Niño: "Nos desmayamos". Guía: “¿Qué } \\
\text { pasaría si al rinoceronte le quitan sus cuernos?" } \\
\text { Niño: "Se podría morir porque esa es la defensa" }\end{array}$ \\
\hline 5 & Explicación & $\begin{array}{l}\text { Verbalizaciones que establecen } \\
\text { relaciones causales sobre un } \\
\text { fenómeno. }\end{array}$ & $\begin{array}{l}\text { Guía: “¿Por qué creen que las tortugas pueden } \\
\text { aguantar varios días sin comer?” Niño: "Como las } \\
\text { tortugas son muy lentas no gastan mucha energía" }\end{array}$ \\
\hline
\end{tabular}

La codificación de las verbalizaciones de los guías y niños visitantes fue realizada por medio del software gratuito MediaCoder 2009 versión 1.1, definido como un programa para el registro en tiempo real de comportamientos almacenados en medios digitales (Bos y Steenbeek 2009). La confiabilidad de las codificaciones fue estimada por medio del análisis Kappa Cohen para establecer el nivel de acuerdo entre codificadores.

Las verbalizaciones de guías y niños se analizaron mediante estadísticos descriptivos, acompañado de análisis de comparaciones múltiples mediante la prueba de Tukey (Tabla 6).

Tabla 6. Plan de análisis para cada objetivo del estudio.

\begin{tabular}{|l|l|l|}
\hline \multicolumn{1}{|c|}{ Objetivo } & \multicolumn{1}{|c|}{ Recursos } & \multicolumn{1}{c|}{ Análisis } \\
\hline Perfiles de las prácticas de guianza. & Instrumento CLASS (pre y post) & $\begin{array}{l}\text { Análisis Cualitativo: Cambios pre- } \\
\text { post con base en las dimensiones de } \\
\text { análisis CLASS. }\end{array}$ \\
\hline $\begin{array}{l}\text { Verbalizaciones de los guías y } \\
\text { participación de los niños visitantes. }\end{array}$ & $\begin{array}{l}\text { Esquemas de Codificación: } \\
\text { Tipología de preguntas de los guías } \\
\text { y verbalizaciones de los niños } \\
\text { visitantes respectivamente. }\end{array}$ & $\begin{array}{l}\text { Estimación de diferencias } \\
\text { significativas pre -intervención- post } \\
\text { de cada guía y a nivel grupal. } \\
\text { Porcentajes de preguntas abiertas y } \\
\text { cerradas en cada fase. } \\
\text { Identificación de cambio de las } \\
\text { trayectorias de complejidad de } \\
\text { verbalizaciones guías y niños } \\
\text { durante los recorridos. }\end{array}$ \\
\hline
\end{tabular}




\section{Resultados}

\section{Caracterización de los recorridos observados en el Museo FCL}

Desde la fase pre-test hasta la fase post-test, se observaron y registraron 28 recorridos guiados con población infantil y adolescentes. Se obtuvieron 7 vídeos por guía y el promedio de visitantes por recorrido fue de 41 niños entre los 5 y 13 años $\left(\overline{\mathbf{X}}_{\text {edad }}=8\right.$ años). En su mayoría (23 recorridos), corresponden a visitantes de educación primaria (grados $1^{\circ}$ a $5^{\circ}$ ), y sólo 5 recorridos correspondieron a visitantes de básica secundaria (Tabla 7).

Tabla 7. Caracterización de los grupos de niños participantes en los recorridos observados.

\begin{tabular}{|c|c|c|c|c|}
\hline Fase & $\begin{array}{c}\text { Rango grado } \\
\text { escolar }\end{array}$ & $\begin{array}{c}\text { Total \# niños } \\
\text { visitantes }\end{array}$ & $\begin{array}{c}\text { Promedio \# niños } \\
\text { visitantes por } \\
\text { recorrido }\end{array}$ & Promedio edad \\
\hline Pre-test & $1^{\circ}-5^{\circ}$ & 268 & 33 & 8 años \\
\hline Intervención & $1^{\circ}-9^{\circ}$ & 511 & 43 & 7 años \\
\hline Post-test & $1^{\circ}-6^{\circ}$ & 356 & 44 & 8 años \\
\hline Todas las fases & $1^{\circ}-9^{\circ}$ & 1135 & 41 & 4 \\
\hline
\end{tabular}

\section{Análisis de las prácticas de guianza}

Basados en la pauta de observación CLASS (dominio de apoyo instruccional), en este apartado se presenta un análisis cualitativo que compara el desempeño de cada uno de los cuatro guías del museo FCL durante el pre-test y el post-test, con el propósito de evaluar la incidencia de la intervención en el fortalecimiento de sus prácticas de guianza.

Perfil práctica de guianza - guía AJ

La comparación del pre-post para AJ (Figura 5), permitió identificar mejoras en su desempeño. Del pre-test al post-test, AJ pasó de puntuaciones bajas a puntuaciones medias en la mayoría de las dimensiones del apoyo instruccional. En la calidad de retroalimentación se observó un incremento de intercambios entre el guía AJ y los niños visitantes, donde el guía retomó las respuestas de los niños y ocasionalmente las relacionó con los contenidos del recorrido. En diálogo instruccional, se observó un incremento en el uso de preguntas abiertas, escucha activa e indagación por expectativas acerca del museo e ideas previas de los niños visitantes.

En formatos para aprender, AJ presentó un desempeño homogéneo caracterizado por seguir un orden lógico durante la presentación de las exhibiciones, ofrecer anticipaciones sobre el recorrido y explicitar los objetivos de aprendizaje. El incremento a puntajes altos en el posttest 1 se debe a la indagación por las expectativas de los niños visitantes. Con respecto a análisis e indagación, se observó un incremento en la participación de los niños para describir y analizar las exhibiciones, más que centrarse en aspectos memorísticos. Respecto a la comprensión del contenido, AJ incrementó el uso de explicaciones más amplias, al recurrir a ejemplificaciones de procesos más que el uso de definiciones puntuales o nombres. Sin embargo, el desempeño del guía puntuó bajo en el post-test 2 debido a que no ofreció ejemplos y pocas veces indagó por ideas previas de los niños visitantes.

Las prácticas de guianza de AJ en el post-test, sugieren un fortalecimiento de su interés por dar lugar a la participación de los niños, para conocer sus opiniones y brindarles más espacios de participación. 


\section{Perfil práctica de guianza - guía BL}

Al comparar el pre-post para el guía BL (Figura 6), se observa un incremento en el diálogo instruccional, al usar preguntas abiertas y cerradas, además de propiciar la participación de los niños visitantes. Sin embargo, las preguntas no promovieron el uso de habilidades de orden superior en los niños visitantes, por lo cual las puntuaciones de análisis e indagación fueron bajas en todas las observaciones. Respecto a formatos para aprender, en el pre-test y post-test BL realizó presentaciones con un orden lógico. En el post-test utilizó anticipaciones del recorrido y tuvo en cuenta la indagación por expectativas de los niños visitantes. En el post-test 2, BL aumentó el uso de ejemplos como estrategia para la presentación de los contenidos de las exhibiciones.

Para la comprensión del contenido, en el pre-test 2 y en el post-test BL indagó por ideas previas y, adicionalmente, sólo en el post-test integró las temáticas del recorrido con experiencias de los niños visitantes. En la calidad de retroalimentación, BL se caracterizó por emplear interacciones pregunta-respuesta. Mientras que en el pre-test 1, el guía retomó algunas de las respuestas de los niños para indicar si eran correctas o no, en el post-test 2 empleó estrategias para ampliar la información suministrada por los niños visitantes, como la devolución de preguntas.

Las prácticas de guianza de BL sugieren un fortalecimiento de conversaciones distribuidas y mayores intercambios con los niños visitantes. Así mismo, el guía a lo largo del estudio se caracterizó por ofrecer recorridos organizados, relacionados con los contenidos de las exhibiciones.

\section{Perfil práctica de guianza - guía DC}

La comparación del pre-post para el guía DC (Figura 7) muestra poca variación en los puntajes, caracterizándose por puntajes medios. El guía DC implementó estrategias pedagógicas que facilitaron la interacción con los niños visitantes. En la comprensión del contenido, el guía usó ejemplos y explicaciones que tenían el objetivo de crear conexiones entre las exhibiciones y las experiencias cotidianas de los niños visitantes. Con relación al análisis e indagación, el guía utilizó preguntas para incentivar la participación mediante actividades de exploración y observación. El guía DC además fomentó espacios de discusión para que los niños realizaran lluvia de ideas sobre los temas abordados.

Respecto a la calidad de retroalimentación y diálogo instruccional, durante el pre-test 1 (puntaje alto) el guía DC realizó procesos de andamiaje mediante preguntas, lo cual facilitó que los mismos niños llegaran a respuestas más elaboradas. En todas las observaciones, DC retomó las respuestas correctas de los niños y las incorporó a sus explicaciones. El diálogo instruccional de DC, se caracterizó en todas las observaciones por la formulación de preguntas abiertas y cerradas, lo que permitió una constante participación de los niños visitantes. Además, DC mostró disposición de escucha y se identificaron conversaciones distribuidas entre guía y niños. En relación con formatos para aprender, a lo largo de todas las observaciones (pre y post), DC no hizo explícitos los objetivos de aprendizaje, pero realizó anticipaciones sobre los temas a tratar. El guía DC mantuvo la atención de los niños visitantes mediante ejemplos sobre las exhibiciones.

Las prácticas de guianza de DC, reveló un aumento de preguntas abiertas en el post-test, al propiciar espacios de participación donde los niños podían expresar sus ideas y, a su vez, estas eran incluidas dentro de la guianza.

Perfil práctica de guianza - guia CM

En la comparación pre-post del guía CM (Figura 8), se identificó un incremento en las dimensiones de comprensión del contenido y calidad de la retroalimentación, debido al uso diversificado 
del tipo de preguntas abiertas y cerradas. Respecto a formatos para aprender, durante todas las observaciones CM presentó la información con un orden lógico, sin explicitar los objetivos de aprendizaje. En las observaciones pre-test CM logró captar la atención de los niños en diferentes momentos y realizó anticipaciones sobre las exhibiciones.

Respecto al análisis e indagación, el guía puntuó bajo en todas las observaciones, debido a la demanda continua de repeticiones, sin embargo, durante el post-test, los niños visitantes aumentaron su participaron mediante la formulación de preguntas y algunas explicaciones. Finalmente, en diálogo instruccional, CM puntuó bajo debido a la ausencia de discusiones durante el recorrido, sin embargo, en el post-test 1 , se observó en CM un aumento en la formulación de preguntas abiertas y cerradas.

Las prácticas de guianza de CM sugieren una mayor disposición para facilitar la participación de los niños visitantes, al establecer relaciones entre las exhibiciones y su vida cotidiana e indagar por sus ideas previas.

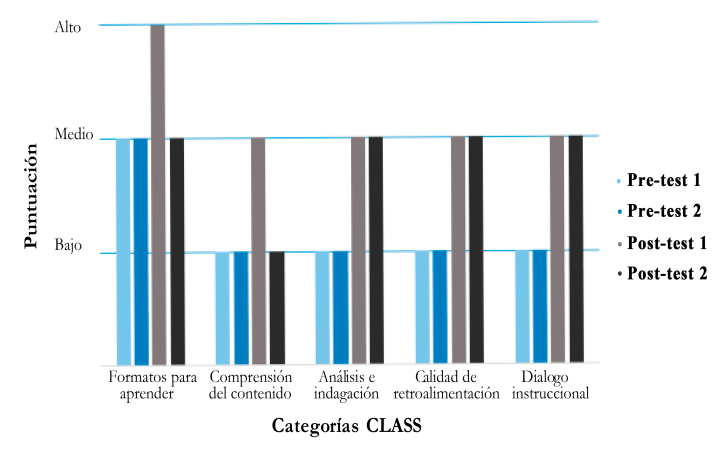

Figura 5. Perfil de guianza-guía AJ

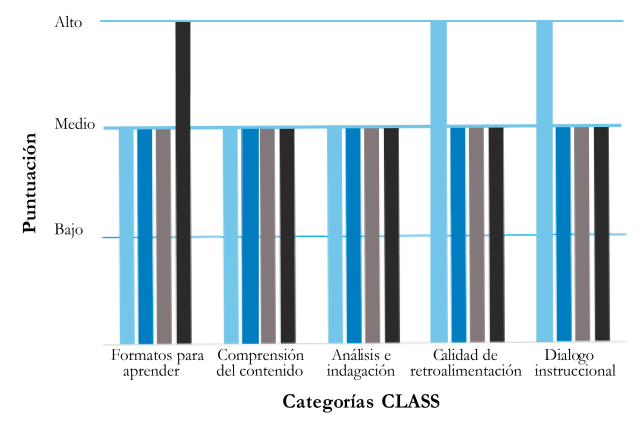

Figura 7. Perfil de guianza-guía DC

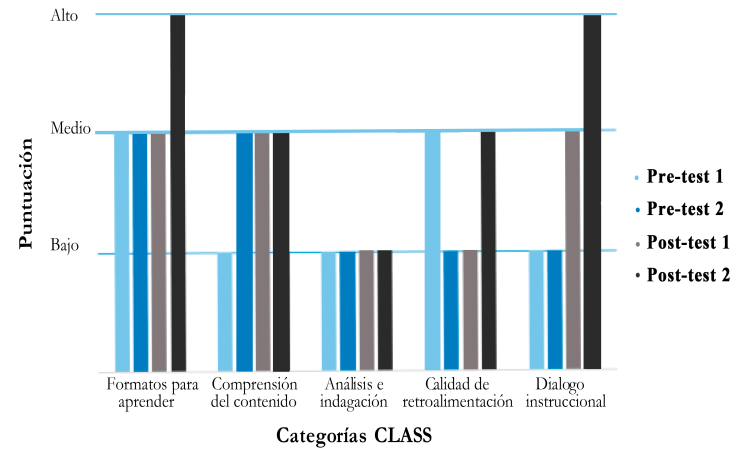

Figura 6. Perfil de guianza-guía BL

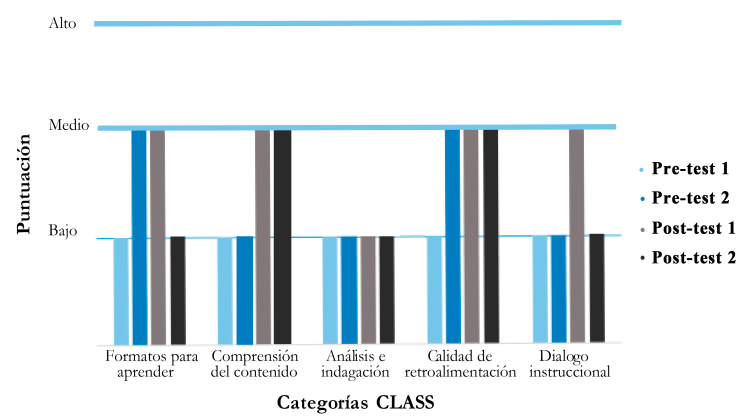

Figura 8. Perfil de guianza-guía CM

Uso de la Indagación por parte de los guías y participación de los grupos de niños visitantes

A partir de la codificación de las verbalizaciones durante todos los recorridos en el museo, se registraron en total 1095 preguntas formuladas por los guías, y 1334 verbalizaciones por parte de los niños visitantes. Con el propósito de establecer la confiabilidad del proceso de codificación de las verbalizaciones, se realizó un análisis inter-observador mediante un coeficiente Kappa de Cohen, donde el $20 \%$ de los vídeos registrados fueron recodificados. Se obtuvo como resultados altos niveles de confiabilidad para cada uno de los esquemas de codificación (Tabla 8).

Tabla 8. Nivel de concordancia inter-observador.

\begin{tabular}{|c|c|c|c|}
\hline Esquema de codificación & Coeficiente Kappa & Nivel concordancia & Porcentaje de acuerdo \\
\hline Tipo de preguntas guías & $0,77(p=, 000)$ & Considerable & $81 \%$ \\
\hline Verbalizaciones niños & $0,84(p=, 000)$ & Casi perfecta & $90 \%$ \\
\hline
\end{tabular}


Para establecer si había diferencias significativas en el nivel de complejidad de las preguntas de los guias, se realizaron comparaciones múltiples a nivel intraindividual y grupal mediante la prueba de Tukey, entre las fases del estudio: pre-test vs intervención, pre-test vs post-test, intervención vs post-test.

Los resultados intraindividuales de esta prueba estadística revelaron diferencias significativas en las comparaciones pre-test vs intervención, y pre-test vs post-test en los casos AJ ( $\mathrm{p}=0.0005$ y $\mathrm{p}=0.0032$, respectivamente) y $\mathrm{CM}$ ( $\mathrm{p}=0.0003$ у $\mathrm{p}=0.0007$, respectivamente). Mientras que en dichos casos, no se encontraron diferencias significativas al comparar las fases intervención vs post-test $(\mathrm{AJ}: \mathrm{p}=0.9826, \mathrm{y} \mathrm{CM}: \mathrm{p}=0.8234)$ a un nivel de confianza de $95 \%$. Estas diferencias significativas permiten inferir que el nivel de complejidad de las preguntas formuladas por cada guía (AJ y $\mathrm{CM}$ ), fue significativamente mayor durante las fases de intervención y post-test que durante el pre-test.

Al comparar estas mismas fases, pre-test vs intervención, pre-test vs post-test e intervención vs post-test, no se encontraron diferencias significativas para los guías BL y DC (guía BL: $\mathrm{p}=0.2181, \mathrm{p}=0.0919$ y $\mathrm{p}=0.7497$, respectivamente) (guía $\mathrm{DC}: \mathrm{p}=0.4683, \mathrm{p}=0.3797$ y $\mathrm{p}=0.9579$, respectivamente).

Los resultados de las comparaciones a nivel grupal, mostraron una diferencia estadísticamente significativa entre las fases pre-test vs post-test $(\mathrm{p}=0.0020)$. Este resultado grupal revela que durante la fase post-test el nivel de la complejidad de las preguntas fue mayor que en la fase pre-test.

Adicionalmente, el análisis descriptivo a nivel grupal acerca de las preguntas empleadas por los guías a lo largo de todas las fases revela: (a) Un aumento en la frecuencia de la formulación de preguntas durante la fase de intervención y post-test, donde la fase de intervención cuenta con el mayor porcentaje de preguntas (49.5\%), lo cual es equivalente a un promedio de 45 preguntas por cada recorrido observado durante la fase de intervención, en contraste con un promedio de 32 y 37 preguntas en las fases pre-test y post-test respectivamente (Tabla 9). Estos resultados sugieren que la intervención fomentó el uso de la indagación por parte de los guías al momento de realizar los recorridos. Sin embargo, esto se vio disminuido en el posttest.

Tabla 9. Cuantificación de las preguntas de los guías durante las fases del estudio.

\begin{tabular}{|l|c|c|c|c|}
\hline Criterio sobre formulación de preguntas & Pre-test & Intervención & Post-test & Total \\
\hline Frecuencia de preguntas & 257 & 542 & 296 & 1095 \\
\hline $\begin{array}{l}\text { Porcentaje preguntas a lo largo de todo el } \\
\text { estudio }\end{array}$ & $23.5 \%$ & $49.5 \%$ & $27 \%$ & $100 \%$ \\
\hline Promedio preguntas por recorrido & 32 & 45 & 37 & 39 \\
\hline
\end{tabular}

Para continuar con el análisis de las preguntas de los guías, la Figura 9 presenta las trayectorias del porcentaje de preguntas abiertas de los guias a lo largo de las observaciones. Si bien el porcentaje de preguntas cerradas durante todas las fases siempre fue mayor al porcentaje de preguntas abiertas, algunos guías pasaron de una baja formulación de preguntas abiertas en el pre-test, a generar ostensiblemente este tipo preguntas en las fases de intervención y post-test. Por ejemplo, el guía AJ pasó de no realizar preguntas abiertas $(0 \%)$ en los pre-test 1 y 2 , a formular este tipo de preguntas hasta en un 58\% (Int-2) durante la intervención, para luego mantenerse en un rango de $38 \%$ a $48 \%$ en el post-test. 


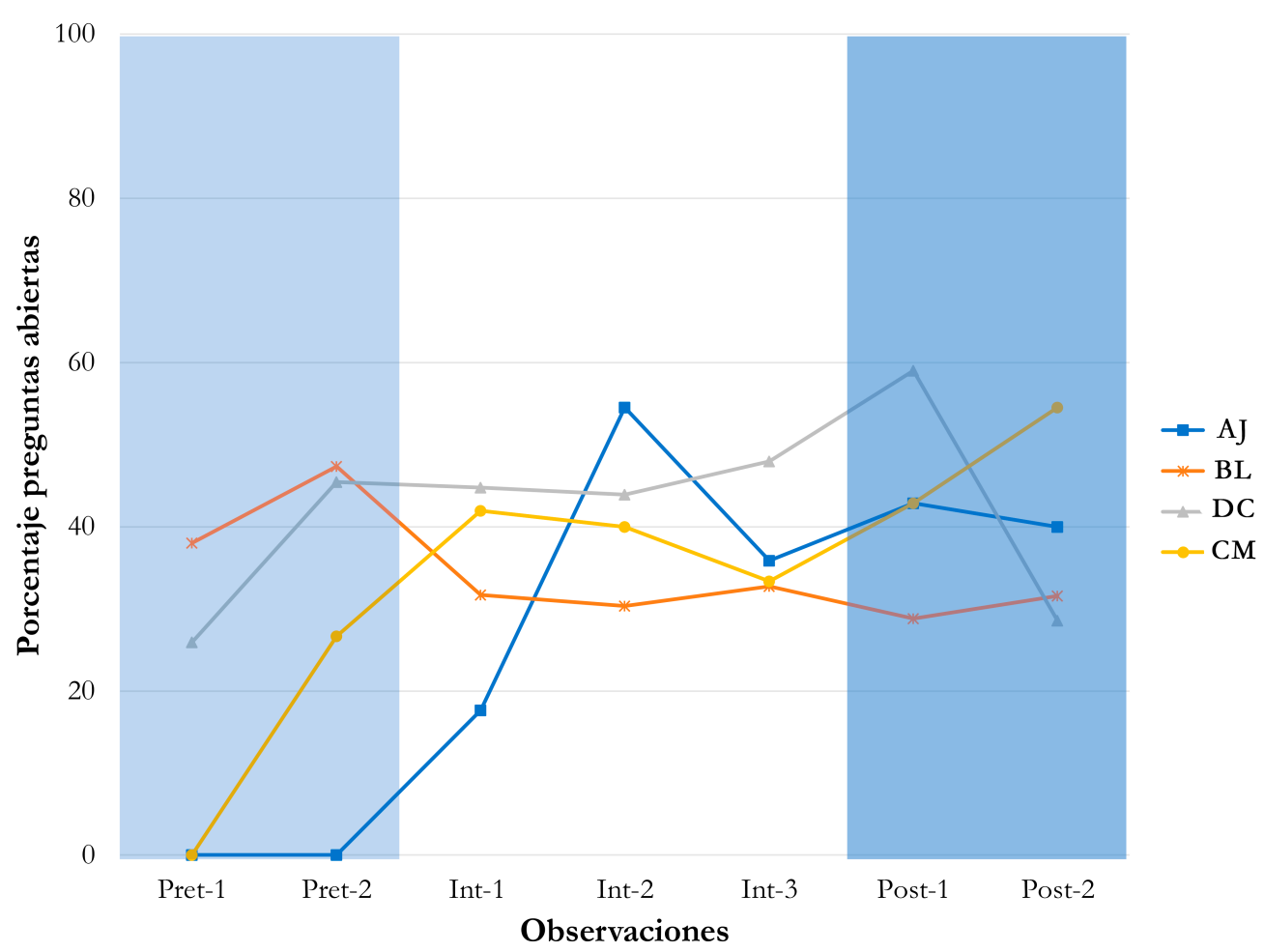

Figura 9. Porcentaje de preguntas abiertas por guía a lo largo de las 7 observaciones.

Con relación a las verbalizaciones de los niños visitantes del museo, la Figura 10 presenta los porcentajes del tipo de verbalizaciones a lo largo de las fases del estudio. Durante la intervención se encontró una reducción del uso de preguntas cerradas y abiertas, y un aumento en el tipo de verbalizaciones que dan cuenta del uso de habilidades de pensamiento científico (descripciones, predicciones y explicaciones). Entre ellas, se destaca el aumento progresivo en el uso de descripciones. En el caso de las predicciones y explicaciones, solo se da un aumento en la fase de intervención, el cual disminuye durante el post-test.

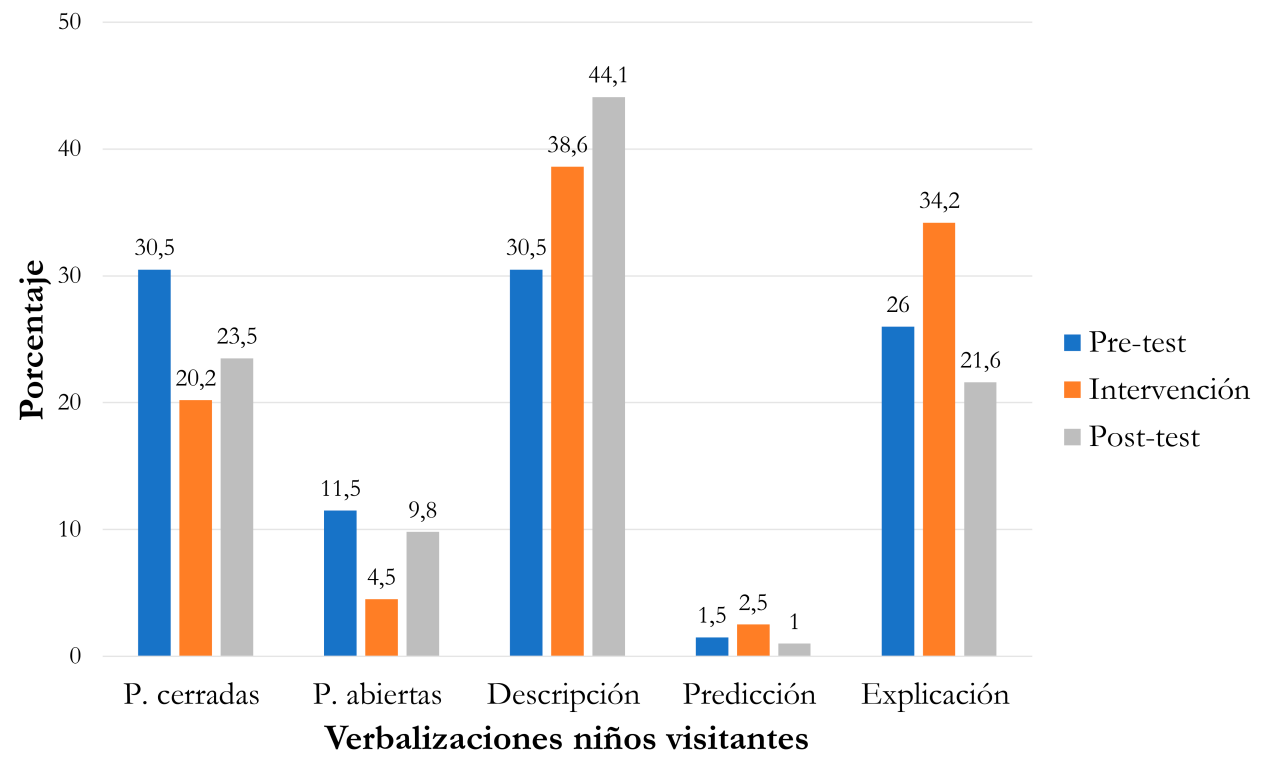

Figura 10. Porcentaje de verbalizaciones de niños visitantes en cada fase del estudio. 
Para finalizar, a continuación se comparan los niveles de complejidad de las verbalizaciones de cada guía (Figura izquierda) y de los niños visitantes (Figura derecha) durante los recorridos observados.

Las verbalizaciones del guía AJ y los niños visitantes del museo, (Figura 11a y 11b), revelan inicialmente bajos niveles de complejidad que luego aumentan en la fase de intervención, debido al mayor porcentaje de preguntas abiertas del guía, con las cuales promueve respuestas más elaboradas por parte de los niños visitantes. Finalmente, en el post-test, el nivel de complejidad de las verbalizaciones de ambos disminuye.

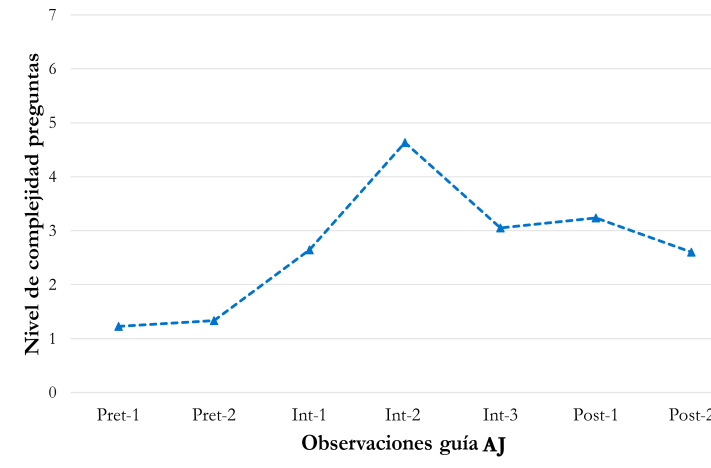

Figura 11a. Complejidad de las preguntas guía AJ.

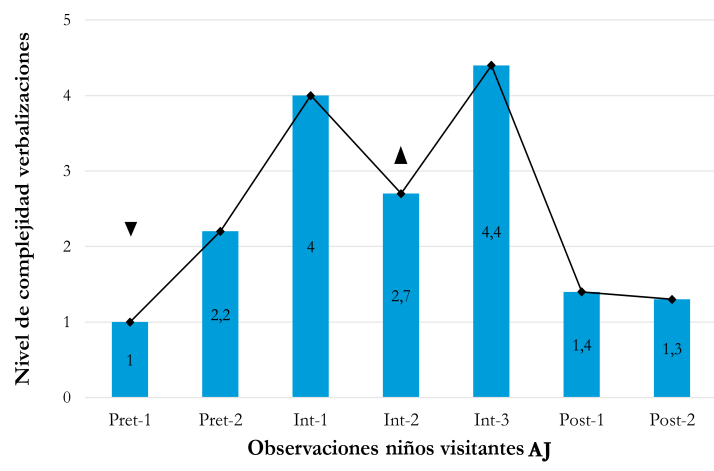

Figura 11b. Complejidad de las verbalizaciones de los niños visitantes con la guianza de AJ.

Nota: $\boldsymbol{\Delta}=$ Máximo Figura 11a. $\boldsymbol{\nabla}=$ Mínimo Figura 11a.

Las verbalizaciones del guia BL y los niños visitantes del museo (Figuras 12a y 12b), muestran niveles medios de complejidad de las pregunta del guía en todas las observaciones (puntajes 3 y 4), y puntajes medio-bajos (puntajes 2 y 4 ) para las verbalizaciones de los niños visitantes. En este caso, no se evidencia una correspondencia entre los niveles de complejidad de las verbalizaciones entre guía y niños.

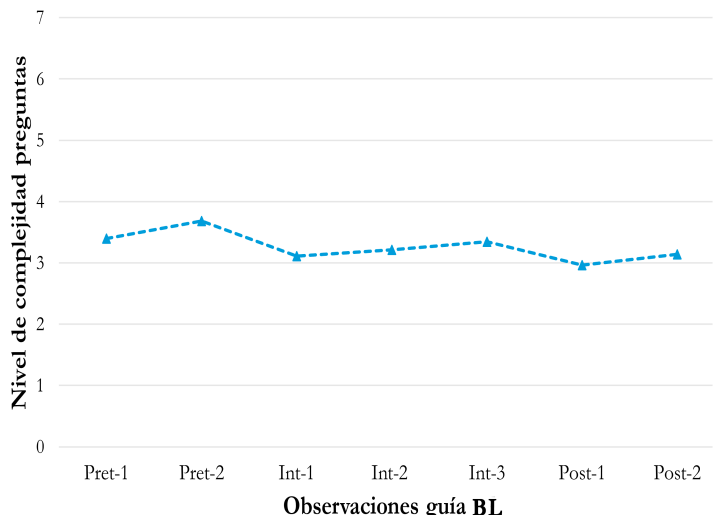

Figura 12a. Complejidad de las preguntas guía BL.

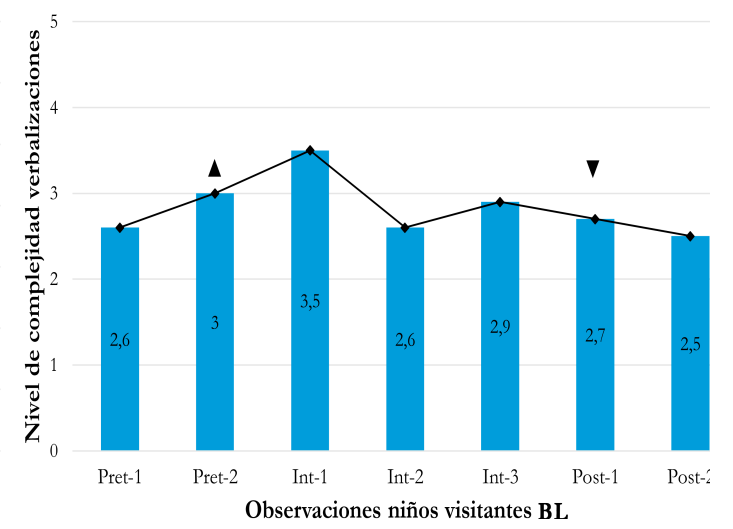

Figura 12b. Complejidad de las verbalizaciones de los niños visitantes con la guianza de BL.

Nota: $\boldsymbol{\Delta}=$ Máximo Figura 12a.

$\boldsymbol{\nabla}=$ Mínimo Figura 12a.

Los niveles de complejidad de las verbalizaciones del guía $D C$ y los niños visitantes del museo (Figura 13a y 13b), muestran trayectorias poco variables. El guía DC mantiene los promedios más altos del nivel de complejidad de las preguntas y obtiene verbalizaciones de alta complejidad por parte de los grupos de niños visitantes. Mediante el uso de preguntas abiertas logra promover en los niños visitantes diversas verbalizaciones (descripciones, predicciones y explicaciones). 


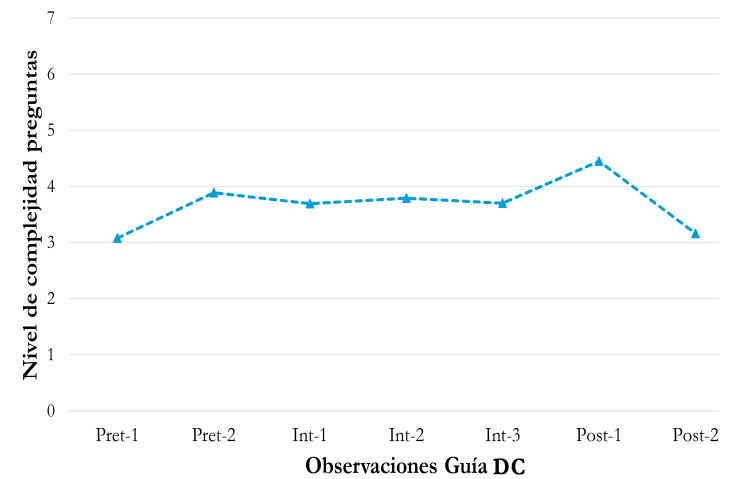

Figura 13a. Complejidad de las preguntas guía DC.

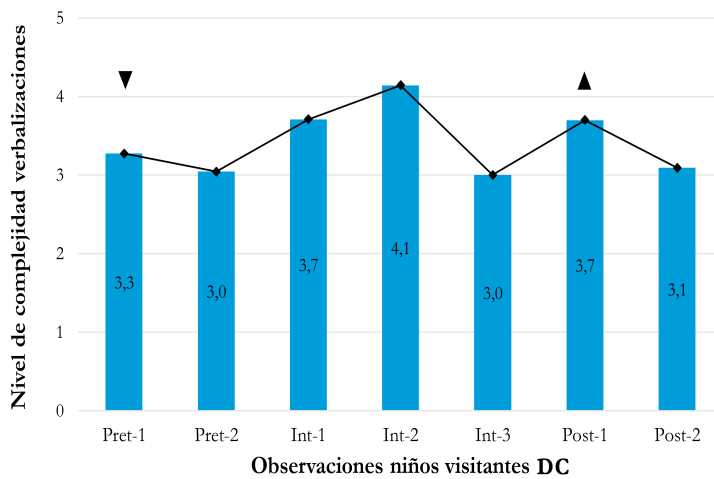

Figura 13b. Complejidad de las verbalizaciones de los niños visitantes con la guianza de DC.

Nota: $\boldsymbol{\Delta}=$ Máximo Figura 13 a.

$\boldsymbol{\nabla}=$ Mínimo Figura 13a.

Las verbalizaciones del guía CM y los niños visitantes del museo (Figura 14a y 14b), muestran una correspondencia en el post-2 respecto al nivel más alto de complejidad tanto en las preguntas del guía $C M$, como en las verbalizaciones de los niños visitantes. Caracterizado por un mayor uso de preguntas abiertas del guía y verbalizaciones más elaboradas por parte de los niños.

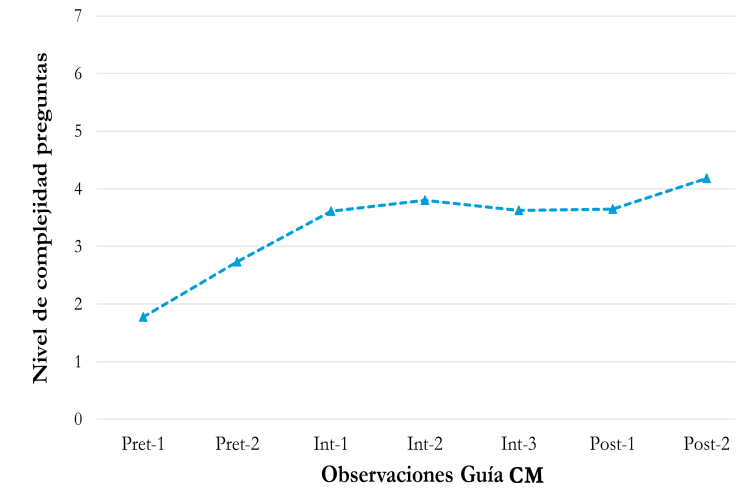

Figura 14a. Complejidad de las preguntas guía CM.

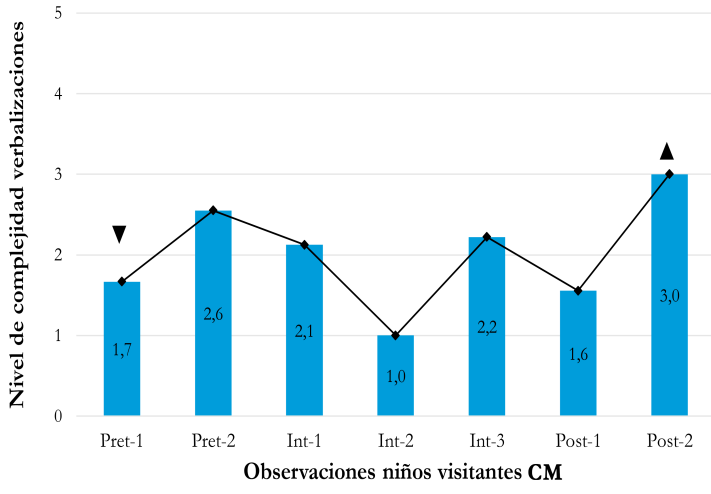

Figura 14b. Complejidad de las verbalizaciones de los niños visitantes con la guianza de CM.

Nota: $\boldsymbol{\Delta}=$ Máximo Figura 14a. $\boldsymbol{\nabla}=$ Mínimo Figura 14a.

\section{Conclusiones}

El presente estudio tuvo como propósito caracterizar y fortalecer las prácticas de guianza en el Museo FCL, a partir de un proceso de intervención centrado en la indagación. Durante el estudio se observó que los cuatro guías del museo dieron lugar a una mayor participación de los niños visitantes en los recorridos, al considerarlos como eje central de sus prácticas de guianza. De modo que el guía, más que un experto que ofrece información de las exhibiciones, se consideró un facilitador de los procesos de aprendizaje en el museo. Es así como el uso de metodologías basadas en indagación involucró a los guías en diversas formas de interacción con los visitantes, donde las preguntas abiertas promovieron procesos de andamiaje.

Las preguntas formuladas por los guías, especialmente en la fase pre-test, corroboran los hallazgos de investigaciones previas en las cuales se identifican tendencias de los agentes educativos (guías en nuestro caso), a usar en mayor medida preguntas cerradas (Lee y Kinzie 2011; Çakır y Cengiz 2016). Adicionalmente, en nuestro estudio se encontró que guías con menor tiempo de experiencia se muestran más dispuestos a implementar el uso de preguntas 
abiertas en comparación con los que contaban con mayor tiempo de experiencia. Estos resultados son semejantes a los hallados por Çakır y Cengiz (2016), en un estudio de prácticas pedagógicas de docentes.

Con respecto al uso de la indagación por parte de los guías, y la participación de los niños visitantes, se encontró evidencia relacionada con los resultados de otros estudios que señalan una relación entre el nivel de complejidad de las preguntas de los guías y el nivel de complejidad de las verbalizaciones resultantes de los niños (Ver Briceño y Tafur 2011; Bamberger y Tal 2007; Lee y Kinzie, 2012; Rivera, Girolametto, Greenberg y Weitzman 2005). En nuestro estudio, altos niveles de complejidad de las preguntas de los guías, se relacionan con altos niveles de complejidad en las verbalizaciones de los niños visitantes del museo.

De modo similar a lo argumentado por Çakır y Cengiz (2016) sobre prácticas pedagógicas, nuestro estudio revela cómo las prácticas de guianza en las que se hace uso de preguntas abiertas, fomentan las habilidades de pensamiento científico de los niños al indagar por sus ideas previas y comprensiones acerca de los recorridos. De esta manera, se reconoce al museo como un espacio propicio para incentivar el aprendizaje más allá de la contemplación, optando por estrategias de indagación como herramientas para involucrar a los niños visitantes con los contenidos de las exhibiciones, fomentando el diálogo y la participación.

Estos hallazgos destacan la necesidad de adaptar las prácticas de guianza a las perspectivas de los niños (y jóvenes) visitantes, donde se reconozca todo su potencial de habilidades de pensamiento y curiosidad espontánea. Lo cual permite que los guías puedan ir más allá del uso de narrativas factuales y manejo memorístico, para concebir a los niños (y jóvenes) visitantes del museo como aprendices activos, con los cuales el proceso de guianza toma lugar mediante una interacción recíproca, en la cual tanto experto como aprendiz se retroalimentan mutuamente.

Respecto a las limitaciones del estudio, se encuentra la restricción de las características del museo, que por su tamaño solo cuenta con una cantidad reducida de guías vinculados, siendo este el único museo de ciencias naturales en la ciudad donde se llevó a cabo el estudio. Por otra parte, teniendo en cuenta el valor del trabajo interdisciplinario, estudios futuros pueden considerar la necesidad de vincular a investigadores, educadores y guías para el diseño de actividades significativas que propicien el aprendizaje en el museo, que permita co-crear ambientes de aprendizaje donde los niños sean bienvenidos. Este propósito consistiría en generar programas especializados para niños y jóvenes que permitan despertar su interés por las ciencias. Igualmente, resulta importante promover el desarrollo de procesos de formación de guías (ver Licontti y Pellegrini 2015), donde el uso de estrategias de intervención como el video-feedback constituye una herramienta potente para fortalecer las habilidades pedagógicas mediante la retroalimentación sobre su desempeño en tiempo real. (ver van Vondel et al. 2017b; Wetzels 2015).

\section{Agradecimientos}

Los autores agradecen al Museo Departamental de Ciencias Naturales Federico Carlos Lehmann de CaliColombia, a los guías y a los niños participantes. Igualmente, al psicólogo Sebastián Salazar Rendón quien hizo parte del equipo investigativo.

\section{Referencias}

Aguirre C. (Ed) (2013) El museo y la escuela: Conexiones, integraciones, complementos. El museo y la escuela. Conversaciones de complemento, Sello Explora-Parque Explora, Medellín, Colombia, 113-130. 
Ampartzaki M., Kypriotaki M., Voreadou C., Dardioti A., Stathi, I. (2013) Communities of practice and participatory action research: the formation of a synergy for the development of museum programmes for early childhood. Educational Action Research 21(1), 4-27.

Ash, D., Klein, C. (2000) Inquiry in the Informal Learning Environment. En Van Zee, E. H. (Ed.), Inquiring into inquiry: learning and teaching in science (pp. 216-240). American Association for the Advancement of Science.

Akamca G., Yildirim R., Ellez A. (2017) An alternative educational method in early childhood: Museum education. Educational Research and Reviews 12(14), 688-694.

Bamberger Y., y Tal T. (2007) Learning in a personal context: Levels of choice in a free choice learning environment in science and natural history museums. Science Education 91(1), 75-95.

Bell J., Falk J., Hughes R., Hunt G., Parrish J., Ruffin M., Troxel, G. (2016) Informal STEM education: Resources for outreach, engagement and broader impacts. Science Education (CAISE).

Bos J., Steenbeek H. (2009) Mediacoder: software voor het coderen van video-en audiomaterialen. Groningen: Internal publication. IDP Department, University of Groningen.

Briceño J., y Tafur M. (2011) Caracterización del diálogo guía-estudiante en un museo interactivo de ciencias y tecnología de Bogotá (Colombia). Revista electrónica de enseñanza de las ciencias 10(2), 289 - 306.

Çakır H., Cengiz O. (2016) The Use of Open Ended versus Closed Ended Questions in Turkish Classrooms. Open Journal of Modern Linguistics 6(02), 60.

Eberbach C., Crowley K. (2017) From seeing to observing: How parents and children learn to see science in a botanical garden. Journal of the Learning Sciences 26(4), 608-642.

Falk J., Storksdieck M., Dierking, L. (2007) Investigating public science interest and understanding: evidence for the importance of free-choice learning. Public Understanding of Science 16(4), 455-469.

Furman M. (2016) Educar mentes curiosas: la formación del pensamiento científico y tecnológico en la infancia: documento básico, XI Foro Latinoamericano de Educación. Santillana.

Galvis C., Concha V. (2018) Aportes para la comprensión de la Curiosidad Científica en niños pequeños: una réplica conceptual (tesis de pregrado). Universidad del Valle, Cali, Colombia.

Glick M., Samarapungavan A. (2008) Wolves are beautiful and proud: Science learning from a school field trip. Journal of Museum Education 33(2), 199-207.

Guevara M. (2015) Peer Interaction and Scientific Reasoning Processes in Preschoolers: An intra-individual approach [Groningen].

Guevara M., van Dijk M., van Geert P. (2016) Microdevelopment of peer interactions and scientific reasoning in young children. Infancia y Aprendizaje 39(4), 727-771.

Guisasola J. y Morentin M. (2005) Museos de ciencias y aprendizaje de las ciencias: una relación compleja. Alambique: Didáctica de las Ciencias Experimentales 12(43), 58-66. 
Hohenstein J., Tran L. (2007) Use of questions in exhibit labels to generate explanatory conversation among science museum visitors. International Journal of Science Education 29(12), 1557-1580.

Kuhn D. (2011) What is scientific thinking and how does it develop?. The Wiley-Blackwell Handbook of Childhood Cognitive Development, 497-523.

Lee Y., Kinzie M. (2011) Teacher question and student response with regard to cognition and language use. Instructional Science 40(6), 857-874.

Li S., Arshad M. (2013) Verbal interaction in chemistry secondary school classroom. Jurnal teknologi (social sciences) 66(1), $21-26$.

Licontti M., Pellegrini N. (2015) Programa de formación para los guías de sala del museo de ciencias de Caracas. Revista de investigación 39(85), 243-257.

Loyens S., Rikers R. (2011) Instruction based on inquiry. Handbook of research on learning and instruction, 361-381.

Martínez M., Godoy F., Treviño E., Varas L., Fajardo G. (2018) ¿Qué nos revelan los instrumentos de observación de aula sobre clases de matemática en escuelas con trayectoria de mejoramiento? São Paulo, Brasil: Educação e Pesquisa 44.

Morentin P., Guisasola A. (2014) La visita a un museo de ciencias en la formación inicial del profesorado de Educación Primaria. Revista Eureka sobre Enseñanza y Divulgación de las Ciencias 11(3), 364-380.

Morris B., Croker S., Masnick A., Zimmerman C. (2012) The emergence of scientific reasoning. In Current topics in children's learning and cognition. IntechOpen. 61-82.

Rivera C., Girolametto L., Greenberg J., Weitzman E. (2005) Children's responses to educators' questions in day care play groups. American Journal of Speech-Language Pathology 14(1), 14-26.

Roldán A., Ulloa D., Vargas L., Chura Z., Pacheco L. (2017) Comparación entre recorridos guiados tradicionales e indagatorios en el Museo Nacional de Historia Natural, La Paz Bolivia. Revista Eureka sobre Enseñanza y Divulgación de las Ciencias 14 (2), 367384.

Ordoñez O. (2020) El desarrollo temprano de la curiosidad científica: Por una pedagogía de la indagación en la educación inicial.

Penning H., van Tartwijk J., Wubbels T., Claessens L., van der Want A., Brekelmans M. (2014) Real-time teacher-students interactions: A dynamic systems approach. Teaching and teacher education, 37, $183-193$.

Pérez Y., Chamizo J. (2011) Los museos: un instrumento para el Aprendizaje Basado en Problemas (ABP). Revista Eureka sobre enseñanza y divulgación de las ciencias 8 (3), 312-322

Perinat A., Lalueza J., Sadurní M. (2007) Psicología del desarrollo: un enfoque sistémico. Barcelona, España: Editorial UOC (Vol. 83).

Pianta R., Hamre B., Mintz S. (2012) Classroom Assessment Scoring System. Upper Elementary Manual (Treviño y colaboradores, trad.). Teachstone.

Sacco K., Falk J., Bell J. (2014) Informal Science Education: Lifelong, Life-Wide, Life-Deep. PLoS Biol 12(11), e1001986. 
Sánchez M. (2013) Museos de ciencias, escuelas y profesorado, una relación a revisarse. Revista Eureka sobre Enseñanza y Divulgación de las Ciencias 10(3), 377-393.

Siegler R., Crowley K. (1991) The microgenetic method: A direct means for studying cognitive development. American Psychologist 46(6), 606.

Silveira F. (2016) Efectos de la visita a un museo interactivo de ciencias en la educación formal. INNOTEC Gestión, (7 ene-dic), 64-71.

Smart J., Marshall J. (2013) Interactions between classroom discourse, teacher questioning, and student cognitive engagement in middle school science. Journal of Science Teacher Education 24(2), 249-267.

Steenbeek H., van Geert P. (2013) The emergence of learning-teaching trajectories in education: A complex dynamic systems approach. Nonlinear Dynamics. Psychology and Life Sciences 17(2), 233-267.

Stocklmayer S., Rennie J., Gilbert K. (2010) The roles of the formal and informal sectors in the provision of effective science education. Studies in Science Education 46(1), 1-44.

van Geert P. (2011) The contribution of complex dynamic systems to development. Child Development Perspectives 5(4), 273-278.

van Schijndel T., Raijmakers M. (2016) Parent explanation and preschoolers' exploratory behavior and learning in a shadow exhibition. Science Education 100(1), 153-178.

van Vondel S., Steenbeek H., van Dijk M., van Geert P. (2017a) Ask, don't tell; A complex dynamic systems approach to improving science education by focusing on the coconstruction of scientific understanding. Teaching and Teacher Education 63, 243-253.

van Vondel S., Steenbeek H., van Dijk M., Van Geert P. (2017b) The Effects of Video Feedback Coaching for Teachers on Scientific Knowledge of Primary Students. Groningen: Springer, 48(2), 301-324.

Wetzels A. (2015) Curious minds in the classroom: the influence of video feedback coaching for teachers in science and technology lessons (Dissertation). University of Groningen. 